\title{
Adsorption Isotherm Of Separation Of Nicotine In Tobacco Products By Low Cost Adsorbents
}

\author{
Zamzam Basher*, A.K.Gupta and Amit Chattre \\ *PhD. Student, Professor, Associate Professor \\ Department of Chemistry, Faculty of Basic Science, SHIATS ,Allahabad-211007(U.P.)
}

\begin{abstract}
The ability of coconut fibre, saw dust and tea waste to adsorb nicotine, from aqueous solutions has been investigated through batch experiments. The amount of nicotine adsorption was found to be dependent on adsorbent dosage and contact time. All experiments were carried out at a constant temperature of $30^{\circ} \mathrm{C}$. The experimental isotherm were analyzed using the Langmuir and Freundlich's equations. The experiments showed that higher removal rate was shown by coconut fibre than saw dust and tea waste.
\end{abstract}

Keywords: Nicotine, Adsorbent, Adsorption isotherms, Removal.

\section{Introduction}

Nicotine, 3-(1-methyl-2-pyrrolidinyl) pyridine [1]is a colourless, pale yellow, hygroscopic oily liquid present in the leaves of Nicotiana tabacum. It is one of the highly toxic chemicals belonging to the tobacco alkaloids [1]. Several gas chromatographic techniques have been described for the determination of nicotine [25]. The most commonly used techniques involve solvent extraction followed by Gas chromatographic-Mass spectrometric analysis[6-8] or Liquid chromatography (LC) with Ultra-Violet Absorbance detection [9]. Davis [10] described a method for the determination of nicotine and cotinine in plasma after a single extraction procedure of Gas Chromatography using nitrogen selective detection.

Moore et al. [11] used the Electron Capture Detector (ECD)for the determination of nicotine after chemical derivatization using heptafluorobutyric anhydride. A very sensitive and selective LC method has been developed for nicotine and $\mathrm{N}$-methylnicotinium ion at the pictogram level using electrochemical detection [12]. Portable ion mobility spectrometers have been used for the detection of low levels of nicotine. The method was applied to the assessment of nicotine content of air and on the breath of smokers and non-smokers [13]. Very few spectrophotometric methods are available for the determination of nicotine. Smith and Cooke[14] used diethylthiobarbituric acid as a spectrophotometric reagent for tobacco alkaloids. The absorbance was measured at $536 \mathrm{~nm}$ and the method was applied to the determination of nicotine in urine samples. Another spectrophotometric method has recently been described [15], based on the bromination of nicotine to form dibromo nicotine which reacts with potassium iodide in the presence of starch to form a water-soluble blue complex, measurable at $580 \mathrm{~nm}$. The method was applied to the determination of total alkaloids and nicotine in tobacco leaves, cigarette smoke and biological samples .In this paper, a simple, new spectrophotometric method is described for the determination of nicotine. The method is based on the reaction of nicotine with adsorbing solution $(0.01 \mathrm{NaOH}$ in $10 \%$ ethanol $)$. The distillate of nicotine extract treated with different low-cost adsorbent and acidified with $0.05 \mathrm{~N}$ hydrochloric acid, representing a known sample weight, is made to volume and diluted with acidified water, the amount of nicotine was determinated by spectral densities , absorbed at wave length $259 \mathrm{~nm}$. The method was applied to the determination of nicotine in cigarettes. The objective of this study was to evaluate the feasibility of using the coconut fibre saw dust and tea waste powder for the removal of nicotine from aqueous solution of nicotine extract. The influence of experimental parameter such as contact time and adsorbent dosage were studied.

\section{Experimental}

The adsorbents (coconut fibre, saw dust and tea waste) were collected from locally available waste material , Washed with distilled water and kept in oven, dried at $80^{\circ} \mathrm{C}$ for 24 hours and powdered with grinder. After grounded, the adsorbents particles were sieved and stored into plastic bag.

An aqueous extract of nicotine $(100 \mathrm{mg} / \mathrm{L})$ was prepared by treating solution with four varieties of cigarettes as Gold, Capstan, Gudang garam , and Tradition in solution of $(0.01 \mathrm{~N}$. NaOH in $10 \%$ ethanol). The distillates of nicotine extract were treated with different low-cost adsorbents and acidified with 0.05 $\mathrm{N}$ hydrochloric acid, representing a known sample weight, diluted with acidified water to $100 \mathrm{ml}$. The amount of nicotine was determinated by spectral densities absorbed at wave length $259 \mathrm{~nm}$. In this process of measurement, the absorption, effect of contact time and amount of adsorbents on absorption of final nicotine were studied . 
Adsorption experiments were performed by contacting $2 \mathrm{~g}$ of each adsorbents with $100 \mathrm{ml}$ of an acidified aqueous extract of nicotine at 1, 2, 3, 6 and 8 hour at room temperature $\left(30^{\circ} \mathrm{C}\right)$. The remaining concentration of nicotine in each sample after adsorption at different time intervals were determined by UVVis spectrophotometer, (PD-3000 UV) after filtering the adsorbent with Whatman filter paper No. 40 (to make it adsorbent free). The nicotine concentration retained in the adsorbent phase was calculated according to formula given below:

$$
\mathrm{q}=\left(\mathrm{C}_{\mathrm{i}}-\mathrm{C}_{\mathrm{f}}\right) \mathrm{V} / \mathrm{W}
$$

where $C_{i}$ and $C_{f}$ are the initial and equilibrium concentrations $(\mathrm{mg} / \mathrm{L})$ of nicotine in solution respectively; $V$ is the volume $(\mathrm{ml})$; and $W$ is the mass $(\mathrm{g})$ of the adsorbent.

\section{Results and Discussion}

Adsorption of nicotine was measured at given contact time of four different cigarettes . From Fig 1 to 3 the plot revealed that the rate of percent nicotine removal is higher at the beginning. This is probably due to larger surface area of the adsorbents being available at beginning for the adsorption of nicotine. Most of the nicotine removal was attained after about $360 \mathrm{~min}$ of shaking time in different adsorbents at all concentrations. On increasing the contact time, the increase in nicotine was observed. Most of the nicotine removal was attained by coconut fibre and saw dust, while minimum removal of nicotine was attained by using waste tea leaves. The result showed that the coconut fibre and saw dust had more efficiency than waste tea leaves adsorbents to remove nicotine in cigarettes. Coconut fibre had a higher mechanical strength as adsorbent or withstand higher stretching energy Coconut fibres are reported as most ductile and energy absorbent material has similar findings has been reported by (Majid Ali,2011).

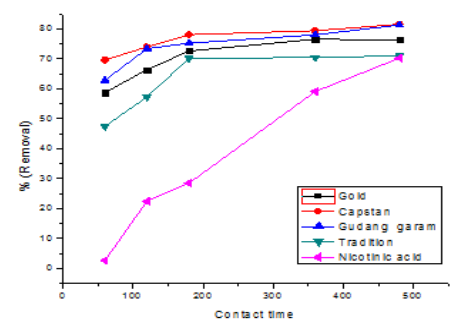

Figure 1: The nicotine adsorption capacity of four cigarettes by Coconut fibre .

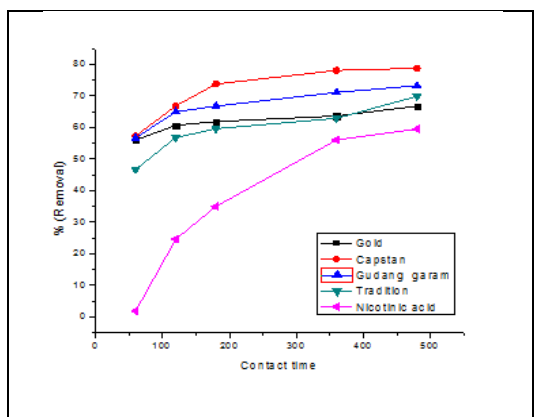

Figure 2: The nicotine adsorption capacity of four cigarettes by Saw dust.

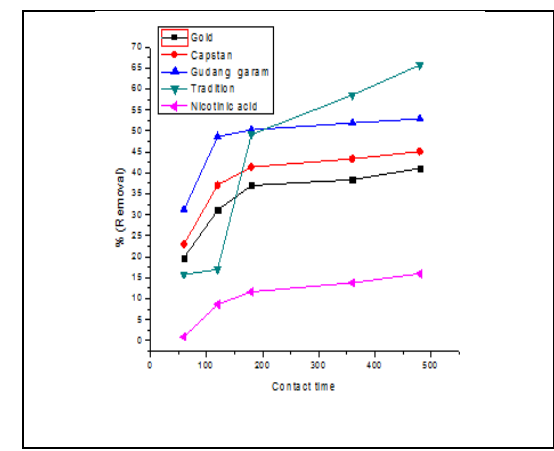

Figure 3: The nicotine adsorption capacity of four cigarettes by Tea waste . 
The effect of the adsorbent dose was studied at room temperature $\left(30^{\circ} \mathrm{C}\right)$ on varying the sorbent amounts of each adsorbent from 0.5 to $2.25 \mathrm{~g}$. Figures from 4 to 6 showed that the adsorption of nicotine increases rapidly with increase in the amount of the adsorbent due to greater availability of the surface area at higher concentration of the adsorbent. A significant increase in uptake was observed when the dose was increased from 0.5 to $2.25 \mathrm{~g}$. Further addition of the adsorbent beyond this did not cause any significant change in the adsorption. This may be due to overlapping of adsorption sites, as a result of overcrowding of adsorbent particles [16]. From the results, it is revealed that within a certain range of nicotine solution, the percentage of nicotine adsorption on the surface of adsorbent is determined by the sorption capacity of the adsorbent.

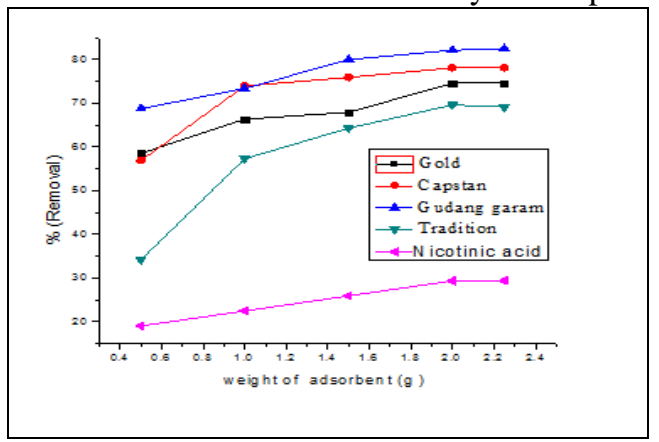

Figure 4:The nicotine adsorption capacity of four cigarettes by Coconut fibre .

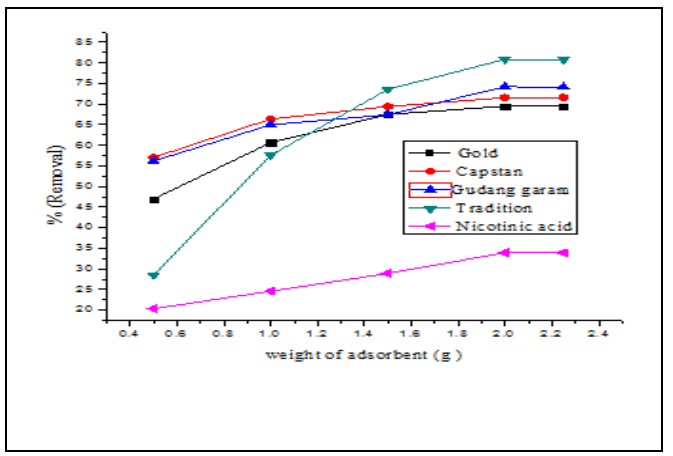

Figure 5:The nicotine adsorption capacity of four cigarettes by Saw dust .

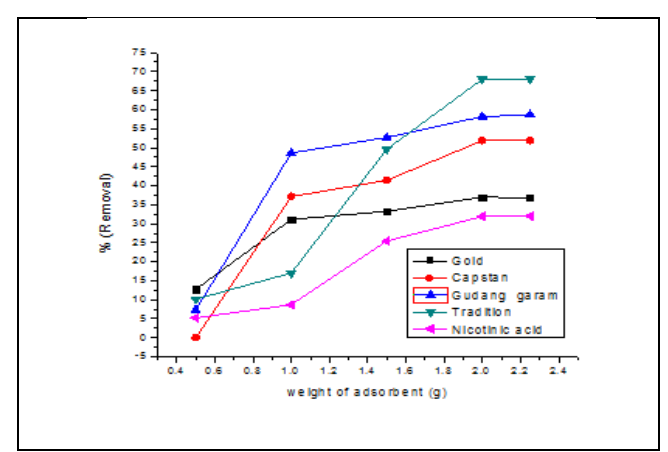

Figure 6:The nicotine adsorption capacity of four cigarettes by Tea waste .

Adsorption isotherms are mathematical models that describe the distribution of the adsorbate species among liquid and adsorbent, based on a set of assumptions that this in are mainly related to the heterogeneity/homogeneity of adsorbents, the type of coverage and possibility of interaction between the adsorbate species. Adsorption data are usually described by adsorption isotherms, such as Langmuir and Freundlich's isotherms. These isotherms relate nicotine uptake per unit mass of adsorbent, $q$, to the equilibrium adsorbate concentration in the bulk fluid phase $C f$.

The Langmuir model $[17,18]$ is based on the assumption that the maximum adsorption occurs when a saturated monolayer of solute molecules is present on the adsorbent surface, the energy of adsorption was constant and there was no migration of adsorbate molecules in the surface plane. The Langmuir isotherm is given by formula:

$\mathrm{q}=\mathrm{q}_{\max } \mathrm{bc}_{\mathrm{f}} / 1+\mathrm{bc}_{\mathrm{f}}$

The constants in the Langmuir isotherm can be determined by plotting $(1 / q)$ versus $(1 / C f)$ and making use of above equation may be rewritten as: 
$1 / \mathrm{q}=1 / \mathrm{c}_{\mathrm{f}}\left(1 / \mathrm{q}_{\max } \mathrm{b}\right)+\left(1 / \mathrm{q}_{\max }\right)$

$q_{\max }$ and $b$ can be determined from the linear plot of $1 / q_{e}$ versus $1 / c_{e}$, where, $q_{\max }$ and $b$ are the Langmuir constants, representing the maximum adsorption capacity for the solid phase loading and the energy constant related to the heat of adsorption respectively. It can be seen from Fig. 7 to 9 that the isotherm data fits the Langmuir equation well $\left(R^{2}=0.739\right)$. The values of $q_{\max }$ and $b$ were determined from the figures and were found to be $2.04,1.88,0.80 \mathrm{mg} / \mathrm{g}$ and $1.63,1.75,0.36 \mathrm{~L} / \mathrm{mg}$, respectively.

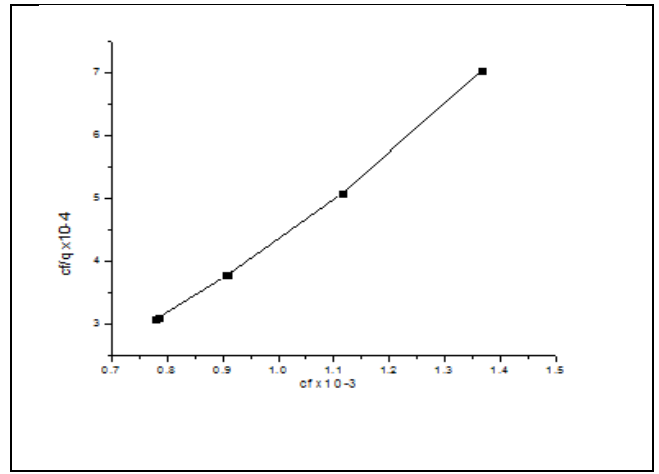

Figure 7: Langmuir's isotherm plot for nicotine in cigarette which adsorbed by Coconut fibre.

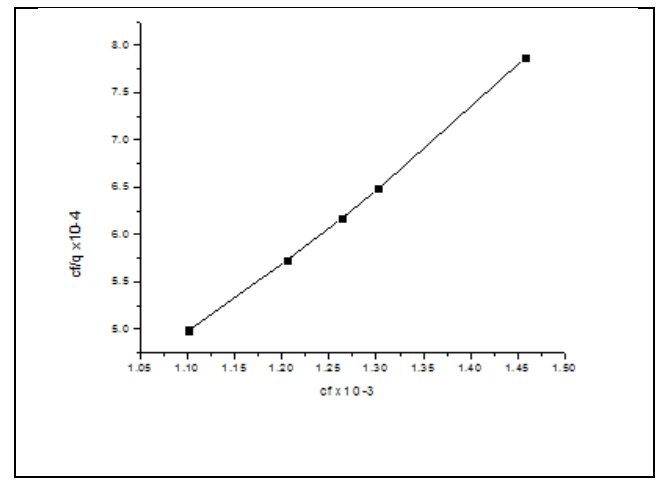

Figure 8: Langmuir's isotherm plot for nicotine in cigarette which adsorbed by Saw dust.

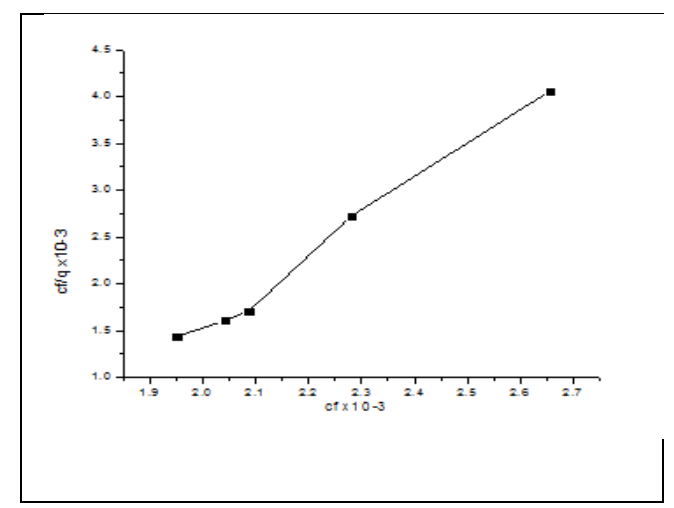

Figure 9: Langmuir's isotherm plot for nicotine in cigarette which adsorbed by Tea waste.

The Freundlich isotherm model $[17,19]$ is an empirical relationship describing the adsorption of solutes from a liquid to a solid surface and assumes that different sites with several adsorption energies that are involved. Freundlich adsorption isotherm is relationship between the amounts of nicotine adsorbed per unit mass of adsorbent $q$, and the concentration of the nicotine at equilibrium $C f$ are as followes: $=\mathrm{k}_{\mathrm{f}} \mathrm{c}_{\mathrm{f}}^{1 / \mathrm{n}}$

Where, $k_{f}$ stands for adsorption capacity and $n$ for adsorption intensity. Logarithmic form of Eq (4) can be written as follows:

$\log q=\log \left(k_{f} c_{f}^{1 / n}\right)$ 
where, $K f$ and $n$ are the Freundlich constants, the characteristics of the system. $K f$ and $n$ are the indicators of the adsorption capacity and adsorption intensity, respectively. The ability of Freundlich model to fit the experimental data was examined. In this case, the plot of $\log C e$ v/s. $\log q$ was employed to generate the intercept value of $K f$ and the slope of $n$. The data portraited in Fig. 10 to 12 envisages that the Freundlich constants $K f$ and $n$ were found to be 2.51,2.24, 1.77 and 2.7, 1.9, 0.1 respectively. The magnitudes of $K f$ and $n$ show easy separation of nicotine from the aqueous solution and indicate favourable adsorption. The intercept $K f$ value is an indication of the adsorption capacity of the adsorbent; the slope $1 / n$ indicates the effect of concentration on the adsorption capacity and represents adsorption intensity. The data given in Table $1, n$ value was found high enough for separation. The Freundlich isotherm is more widely used but provides no information on the monolayer adsorption capacity in contrast to the Langmuir's model. Freundlich isotherm fitted well with the correlation coefficient of 0.729 .

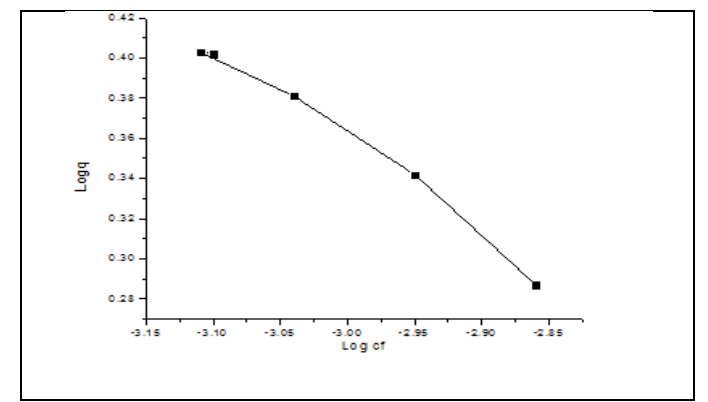

Figure 10:Freundlich's isotherm plot for nicotine in cigarette which adsorbed by Coconut fibre.

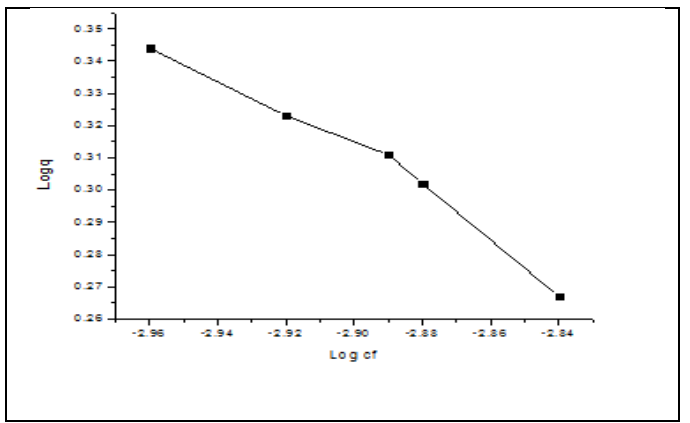

Figure 11:Freundlich's isotherm plot for nicotine in cigarette which adsorbed by Saw dust.

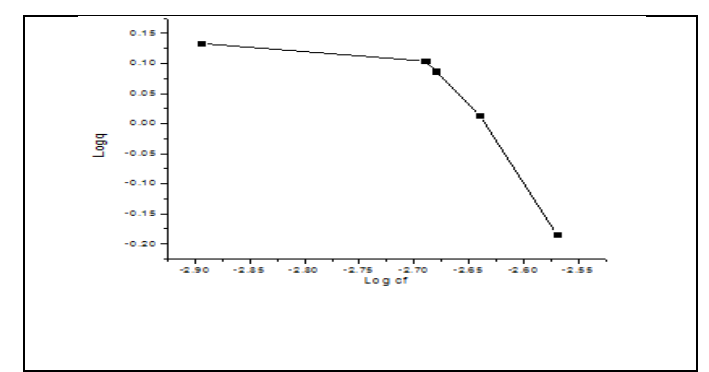

Figure 12:Freundlich's isotherm plot for nicotine in cigarette which adsorbed by Tea waste.

Table 1: Constants of Isotherm Models for Adsorption of Nicotine from aqueous solution of gold cigarette.

$$
\text { Langmuir Freundlich }
$$

\begin{tabular}{lcccc}
\hline Adsorbent & $\mathrm{q}_{\max }$ & $\mathrm{b}$ & $\mathrm{k}$ & $\mathrm{n}$ \\
& & & & \\
\hline Coconut fibre & 2.04 & 1.63 & 2.51 & 2.7 \\
Saw dust & 1.88 & 1.75 & 2.24 & 1.9 \\
Tea waste & 0.80 & 0.36 & 1.77 & 0.1 \\
\hline
\end{tabular}




\section{Conclusions}

The present investigation showed that coconut fibre and saw dust were an effective adsorbents for the removal of nicotine from aqueous solutions of cigarettes . From study, it is observed that adsorption of nicotine is very rapid in the initial stage and later decreases while approaching equilibrium. The percentage removal of nicotine increased with the increase in contact time and adsorbent dosage. The adsorption of nicotine increased rapidly with increase in the amount of the adsorbent, but it became constant at specific amount of adsorbent, any further addition of the adsorbent did not cause significant change in the adsorption. Experimental results are in good agreement with Langmuir's and Freundlich's adsorption isotherm models, and have shown a good fitting to the experimental data .

\section{Reference}

[I] The Merck Index, 11th ed., 1989, p. 1030.

[2] I.E. Burrows, P.J. Corp and G.C. Jackson, REI. Page, Analyst 96 (1971) 81.

[3] A.H. Beckett and E.J. Triggs, Nature 211 (1966) 1415

[4] P.E Isaac and M.J. Nature 236 (1972) 307.

[5] C. Feyerabend, T. Levitt and M.A.H. Russell, J. Pharm. Pharmacol. 27 (1975) 434

[6] I. Dow and K. Hall, J. Chromatogr. 153 (1978) 521.

[7] J.A. Thompson, M.S. Ho and D.R. Petersen, J. Chromatogr. 232 (1982) 53.

[8] P. Grubner, M.W. First and G.L. Huber, Anal. Chern. 52 (1980)1755.

[9] I.D. Watson, J. Chromatogr. 143 (1977) 203.

[10] R.A. Davis, J. Chromatogr. Sci. 24 (1986) 134

[11] J.M. Moore, D.A. Cooper, T.C. Kram and REX. Klein, J.Chromatogr. 645 (1993) 273

[12] S. Mousa, G.R Van Loon, A.A. Houdi and P.A. Crooks, J.Chromatogr. 347 (1985) 405.

[13] E Keenan and M. Cooke, Anal. Proc. Including Anal. Comm. 31(1994) 27.

[14] C.L. Smith and M. Cooke, Analyst 112 (1987) 1515.

[15] M. Rai, K.N. Ramachandran and V.K. Gupta, Analyst 119 (1994)1883.

[16] Namasivayam, C.Prabha, D. and Kumutha, M. (1998). Removal of direct red and acid brilliant blueby adsorption on to banana pith. BioresourceTechnology, 64(1), 77-79.

[17] Agyei, N.M.; Strydom, C.A. and Potgieter, J.H. (2000). An investigation of phosphate ion adsorption from aqueous solution by fly ash and slag. Cem. and Concr. Res., 30(5), 823-826.

[18] Ho, Y.S. and McKay, G. (1999). Competitive sorption of copper and nickel ions from aqueous solution using peat. AdsorptionJournal of the International Adsorption Society, 5(4), 409-417.

[19] Baup, S.; Jaffre, C.; Wolbert, D. and Laplanche, A. (2000). Adsorption of pesticides onto granulatedactivated carbon: determination of surface diffusivities using simple batch experiments. Adsorption, 6(3), 219-228. 